\title{
Frame dependence of form factors in light-front dynamics
}

\author{
Yang $\mathrm{Li}_{1}{ }^{1,2}$ Pieter Maris, ${ }^{2}$ and James P. Vary ${ }^{2}$ \\ ${ }^{1}$ Department of Physics, College of William and Mary, Williamsburg, Virginia 23185, USA \\ ${ }^{2}$ Department of Physics and Astronomy, Iowa State University, Ames, Iowa 50011, USA
}

(Received 10 December 2017; published 29 March 2018)

\begin{abstract}
In light-front dynamics, form factors are traditionally computed with the "good current" $J^{+}$within the Drell-Yan frame $q^{+}=0$. Due to truncations imposed in practical calculations, the from factor may acquire frame dependence, which is often neglected. In this work, we explore the form factors in more general frames, preserving the boost covariance. We find the frame dependence of the elastic form factors for mesons is small in basis light-front holography and related models with two-body Fock space truncation. We suggest to use the difference between form factor results from Drell-Yan frame and the "longitudinal frame" as a metric for the violation of the Lorentz symmetry due to Fock space truncation.
\end{abstract}

DOI: $10.1103 /$ PhysRevD.97.054034

\section{INTRODUCTION}

In quantum field theory, elastic electromagnetic form factors characterize the structure of a bound state system. They generalize the multipole expansion of charge and current density in nonrelativistic quantum mechanics. Formally, they are defined as the Lorentz scalars arising in the Lorentz structure decomposition of the hadron matrix element $\left\langle\psi_{h}(p+q)\left|J^{\mu}(0)\right| \psi_{h}(p)\right\rangle$. If the hadron state vector $\left|\psi_{h}(p)\right\rangle$ is known for arbitrary momentum $p$ of interest, the hadron matrix element can be directly obtained. Light-front wave functions (LFWFs) are boost invariant objects hence are particularly advantageous for this task. LFWFs are the eigenfunctions of the light-front quantized Hamiltonian operator at a fixed light-front time $x^{+} \equiv t+z / c$. See Refs. [1-7] for reviews of this topic.

Although, by definition, form factors are Lorentz invariants, calculations in light-front dynamics are typically done using a specific component $J^{+} \equiv J^{0}+J^{3}$, the "good current," and in a special frame $q^{+}=q^{0}+q^{3}=0$, the DrellYan frame ${ }^{1}$ [8-11]. The main advantage of this combination of current and frame choices is that vacuum pair production/ annihilation is suppressed [10-12]. As a result, parton number is conserved and the matrix element only involves the overlap of LFWFs of the same parton number [see Fig. 1(a)]. Nevertheless, this is not an a priori restriction and other frames and/or components can be used. However, since the parton number is no longer conserved, higher Fock sector

\footnotetext{
${ }^{1}$ Note that it represents infinite many of frames related by lightfront boost transformation.

Published by the American Physical Society under the terms of the Creative Commons Attribution 4.0 International license. Further distribution of this work must maintain attribution to the author(s) and the published article's title, journal citation, and DOI. Funded by SCOAP ${ }^{3}$.
}

wave functions are needed [see Fig. 1(b)]. In most practical calculations, only a finite number of partons can be retained in the Fock space, though exceptions exist, e.g. [13]. Consequently, with truncated Fock sector representations, form factors evaluated in different frames or using different components of the current give different results, implying the loss of Lorentz covariance.

Note that even with the combination of Drell-Yan frame and $J^{+}$, higher Fock sector contributions are present. Neglecting these contributions could also lead to violation of Lorentz symmetry. Since in practical calculations, Fock sector truncation is part of the model, the frame dependence of form factors may be used to as a metric of the violation of Lorentz covariance within the model [14]. This suggestion of a useful metric also applies to other observables. The hope is that, as more Fock sectors are included, the frame dependence may be reduced, as is shown in some specific models [15-17].

The light-front projection of the Bethe-Salpeter amplitude (BSA) provides another insightful perspective. In particular, using perturbatively obtained $\mathrm{BSA}^{2}{ }^{2}$ it was shown that apart from the overlap of LFWFs, a non-LFWF-overlap contribution, known as the Z-diagrams, also emerges ([12,18-20], see Fig. 2). These diagrams are a partial resummation of higher Fock sector contributions. They do not necessarily vanish even in the Drell-Yan frame except for $J^{+}$for scalar theory $[12,18]$. However, beyond perturbation theory, it is not clear to what extent the Z-diagram is large or how to evaluate their contributions, although they can be separately modeled $[21,22]$. Furthermore, modern Bethe-Salpeter equations coupled with the Dyson-Schwinger equations are formulated

\footnotetext{
${ }^{2}$ For these calculations, one can also start directly from lightcone perturbation theory, as it is equivalent to the light-front projection of the covariant perturbation theory.
} 


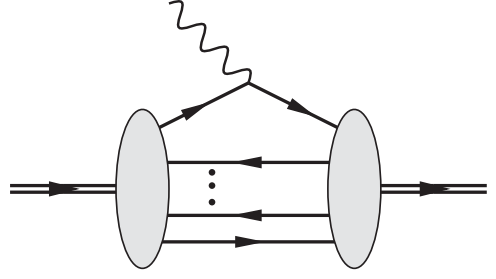

(a) Parton-number-conserving contributions



(b) Parton-number-non-conserving contributions

FIG. 1. LFWF representation of the hadron matrix element. The double-lines represent the hadrons. The solid lines represent the partons. The wavy lines represent the probing photon. The shaded areas represent the LFWFs. These diagrams are ordered by light-front time $x^{+}$, which flows from left to right. The parton-number-nonconserving contributions (b) stem from pair production/annihilation.

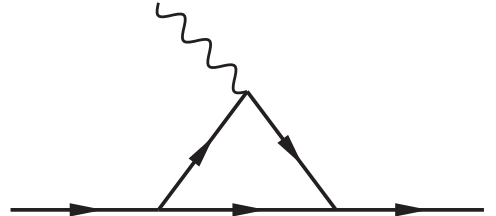

(a) Triangle diagram time ordering a

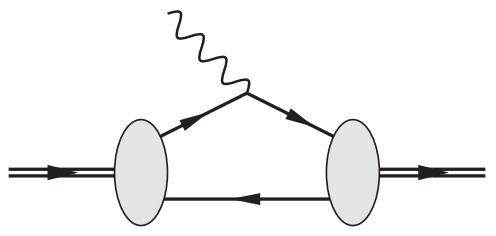

(c) Overlap contributions

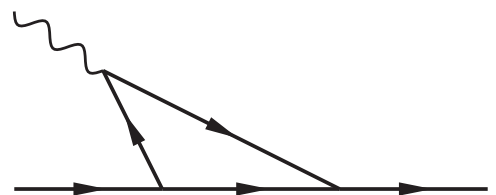

(b) Triangle diagram time ordering $b$

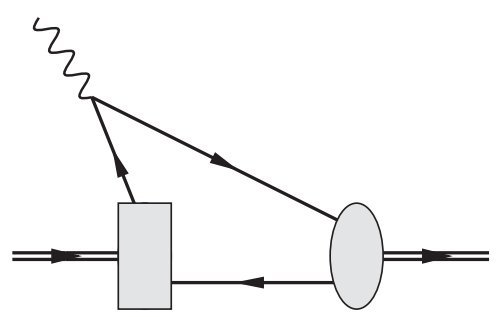

(d) Z-diagram contributions

FIG. 2. Top: The light-front projections of the covariant triangle diagrams. (a) and (b) represent different time ordering. Bottom: The light-front projection of the Bethe-Salpeter amplitude representation of form factors. The shaded ovals represent LFWFs. The rectangle represents a non-LFWF vertex. (c) resembles (a) while (d) resembles (b).

in Euclidean space time. To evaluate the form factors, the current may also have to be consistently dressed [23]. The bridge between the Euclidean Bethe-Salpeter and the Minkowskian light-front approaches is not yet built [24-31].

The dependence of form factors on the current components and on the reference frame are two typical symptoms of the violation of the Lorentz covariance in light-front dynamics. Model independent analysis of current components in the Drell-Yan frame has been performed extensively by Karmanov and collaborators in the covariant formulation of light-front dynamics [6] (cf. [12]). Apart from the formal aspects, the use of other current components for certain observables is more physically justified. For example, in nonrelativistic quantum mechanics, the magnetic moments can only be extracted from 3 -dimensional current density operator $\vec{J}$. While in quantum field theory, relativity allows us to extract magnetic moments from the charge density operator $J^{0}$, or in lightfront dynamics, $\mathrm{J}^{+}$, this procedure requires a proper implementation of Lorentz covariance within the model. In contrast, the current density operator $\vec{J}_{\perp}$, echoing its nonrelativistic counterpart, should provide a more reliable access to magnetic moments, at least in heavy quarkonia [32].

On the other hand, the investigation of the frame dependence in elastic form factors is rare to the best of our knowledge $[12,14,18,19]$. Most studies focus on the transition form factors in the time-like region where the Drell-Yan frame is not applicable $[12,21,33,34]$. This work is intended to fill the gap. In particular, we propose a new parametrization, in which the form factors are expressed as a function of two boost invariants $z, \Delta_{\perp}$ [see Eq. (10)]. If the Lorentz covariance is restored, the dependence is reduced to a single Lorentz invariant $Q^{2}=\left(z^{2} M_{h}^{2}+\Delta_{\perp}^{2}\right) /(1-z)$.

As a concrete example, we scrutinize the frame dependence of the elastic charge form factor of (pseudo-)scalar mesons for heavy quarkonia in a phenomenological model based on 
light-front holographic QCD. In (pseudo-)scalar mesons, the frame and the component dependence largely separate, so that we can focus on the former. We discover that the frame dependence is moderate in heavy quarkonia. We also find the frame dependence can be characterized by the discrepancy between two frames: the Drell-Yan frame and the "longitudinal frame", the meaning of which will be explained later. Finally, we will also comment on the frame dependence in light meson elastic form factors based on calculations with light-front holographic QCD wave functions.

This paper is organized as follows. In Sec. II we present the formalism for computing form factors in a general frame. In Sec. III we apply the formulation to light-front wave functions for heavy quarkonia. We conclude in Sec. IV.

\section{FORM FACTOR IN LIGHT-FRONT DYNAMICS}

The Lorentz decomposition of the matrix element of a (pseudo)scalar meson $h$ is [6],

$$
\begin{aligned}
\Gamma^{\mu}\left(p, p^{\prime} ; \omega\right) & \equiv\left\langle\psi_{h}\left(p^{\prime} ; \omega\right)\left|J^{\mu}(0)\right| \psi_{h}(p ; \omega)\right\rangle \\
& =\left(p+p^{\prime}\right)^{\mu} F\left(z, Q^{2}\right)+\frac{\omega^{\mu}}{\omega \cdot p^{\prime}} S\left(z, Q^{2}\right),
\end{aligned}
$$

where $q_{\mu}=p_{\mu}^{\prime}-p_{\mu}, z=\omega \cdot q / \omega \cdot p^{\prime}, Q^{2}=-q^{2}, \omega$ is a fixed null 4-vector $\left(\omega_{\mu} \omega^{\mu}=0\right)$ indicating the orientation of the quantization surface. For the elastic form factor, $p^{2}=p^{\prime 2}=M_{h}^{2}$. Because the light-front quantized state vector $\left|\psi_{h}\right\rangle$ depends on $\omega,{ }^{3}$ in covariant light-front dynamics (CLFD, [6]), the Lorentz structure of the hadronic matrix element is extended. Similar analysis can be applied to the decay constant as well as the non-local matrix elements, e.g. distribution amplitude and generalized parton distribution. In the case of (pseudo)scalar mesons, apart from the physical form factor $F$, there appears a spurious form factor $S$. Furthermore, the form factors depend on two Lorentz scalars $Q^{2}$ and $z{ }^{4}$ If the Lorentz symmetry is dynamically restored, the spurious form factor $S$ will vanish, as will the dependence of the form factors on $z$.

The emergence of the spurious form factors in CLFD is general. For spin-1/2 hadrons, there are 3 spurious form factors; for spin-1, the number is 8 [6]. These spurious form factors are expected to vanish dynamically when the truncations are lifted. For an ab initio calculation, our best hope is that these form factors are suppressed by powers of $\Lambda_{\mathrm{QCD}} / \Lambda$, where $\Lambda$ is the UV scale associated with the truncation.

In this work, we choose the standard light-front dynamics, i.e. $\omega=\left(\omega^{0}, \vec{\omega}\right)=(1,0,0,-1)$. The light-cone coordinates are defined as $v^{ \pm}=v^{0} \pm v^{3}$, and $\vec{v}_{\perp}=\left(v^{1}, v^{2}\right)$. In this convention, $\omega^{-}=2, \omega^{+}=\omega_{\perp}=0$, and $\omega \cdot v=v^{+}$. Note that this choice does not automatically make the spurious form factor $S$ or the frame dependence disappear. Indeed, the spurious form factor $S$ enters the hadron matrix elements of $J^{-}$, and leads to the violation of the current conservation:

$$
q_{\mu} \Gamma^{\mu}\left(p, p^{\prime} ; \omega\right)=z S\left(z, Q^{2}\right)
$$

The physical form factor $F\left(z, Q^{2}\right)$ can be extracted from either $J^{+}$or $\vec{J}_{\perp}$ :

$\left\langle\psi_{h}\left(p^{\prime} ; \omega\right)\left|J^{+}(0)\right| \psi_{h}(p ; \omega)\right\rangle=\left(p^{+}+p^{\prime+}\right) F\left(z, Q^{2}\right)$,

$\left\langle\psi_{h}\left(p^{\prime} ; \omega\right)\left|\vec{J}_{\perp}(0)\right| \psi_{h}(p ; \omega)\right\rangle=\left(\vec{p}_{\perp}+\vec{p}_{\perp}^{\prime}\right) F\left(z, Q^{2}\right)$.

The current components $J^{+}$and $\vec{J}_{\perp}$ are related by a kinematical boost in the transverse direction:

$$
\begin{aligned}
& \left\langle\psi_{h}\left(p^{++}, \vec{p}_{\perp}^{\prime}+p^{++} \vec{\beta}_{\perp} ; \omega\right)\left|\vec{J}_{\perp}\right| \psi_{h}\left(p^{+}, \vec{p}_{\perp}+p^{+} \vec{\beta}_{\perp} ; \omega\right)\right\rangle \\
& =\left\langle\psi_{h}\left(p^{\prime+}, \vec{p}_{\perp}^{\prime} ; \omega\right)\left|\vec{J}_{\perp}\right| \psi_{h}\left(p^{+}, \vec{p}_{\perp} ; \omega\right)\right\rangle \\
& \quad+\vec{\beta}_{\perp}\left\langle\psi_{h}\left(p^{\prime+}, \vec{p}_{\perp}^{\prime} ; \omega\right)\left|J^{+}\right| \psi_{h}\left(p^{+}, \vec{p}_{\perp} ; \omega\right)\right\rangle .
\end{aligned}
$$

Substituting (3), (4), these two current components lead to the same results for the form factor $F\left(z, Q^{2}\right)$, as expected.

Next, we turn to the frame dependence, i.e. $z$ dependence of the physical charge form factor $F\left(z, Q^{2}\right)$. The meson state vector $\left|\psi_{h}(p, j, \lambda)\right\rangle$ can be expanded in the Fock space,

$$
\begin{aligned}
\left|\psi_{h}(p, j, \lambda)\right\rangle= & \sum_{n} \prod_{i=1}^{n} \sum_{s_{i}} \int \frac{\mathrm{d} x_{i}}{2 x_{i}} \frac{\mathrm{d}^{2} k_{i \perp}}{(2 \pi)^{3}} 2 \delta\left(x_{1}+x_{2}+\cdots+x_{n}\right)(2 \pi)^{3} \delta^{2}\left(\vec{k}_{1 \perp}+\vec{k}_{2 \perp}+\cdots+\vec{k}_{n \perp}\right) \\
& \times \psi_{h / n}\left(\left\{x_{i}, \vec{k}_{i \perp}, s_{i}\right\}\right) c_{s_{1}}^{\dagger}\left(x_{1} p^{+}, \vec{k}_{1 \perp}+x_{1} \vec{p}_{\perp}\right) \times \cdots \times c_{s_{n}}^{\dagger}\left(x_{n} p^{+}, \vec{k}_{n \perp}+x_{n} \vec{p}_{\perp}\right)|0\rangle \\
= & \sum_{s, \bar{s}} \int_{0}^{1} \frac{\mathrm{d} x}{2 x(1-x)} \int \frac{\mathrm{d}^{2} k_{\perp}}{(2 \pi)^{3}} \psi_{s \bar{s} / h}^{(\lambda)}\left(x, \vec{k}_{\perp}\right) \\
& \times b_{s}^{\dagger}\left(x p^{+}, \vec{k}_{\perp}+x \vec{p}_{\perp}\right) d_{\bar{s}}^{\dagger}\left((1-x) p^{+},-\vec{k}_{\perp}+(1-x) \vec{p}_{\perp}\right)|0\rangle+\cdots
\end{aligned}
$$

\footnotetext{
${ }^{3}$ The dependence is always in the form of a ratio, e.g. $\omega^{\mu} / \omega \cdot p$, as there is an additional conformal symmetry, i.e. $\lambda \omega^{\mu}$ and $\omega^{\mu}$ indicate the same quantization surface [6].

${ }^{4} z$ is not a Lorentz invariant, as $\omega$ is a fixed 4-vector.
} 
where $\psi_{h / n}\left(\left\{x_{i}, \vec{k}_{i \perp}, s_{i}\right\}\right)$ are the LFWFs. The dots represent the higher Fock sector contributions. The current operator $J^{\mu}=\bar{\psi} \gamma^{\mu} \psi$, where the quark field operator $\psi$ at $x^{+}=0$ is,

$$
\psi(x)=\left.\sum_{s} \int \frac{\mathrm{d} p^{+} \mathrm{d}^{2} p_{\perp}}{(2 \pi)^{3} 2 p^{+}} \vartheta\left(p^{+}\right)\left[b_{s}\left(p^{+}, \vec{p}_{\perp}\right) u_{s}\left(p^{+}, \vec{p}_{\perp}\right) e^{-\mathrm{i} p \cdot x}+d_{s}^{\dagger}\left(p^{+}, \vec{p}_{\perp}\right) v_{s}\left(p^{+}, \vec{p}_{\perp}\right) e^{+\mathrm{i} p \cdot x}\right]\right|_{x^{+}=0} .
$$

The operators $b$ and $d$ satisfy the standard anticommutation relation,

$$
\left\{b_{s}\left(p^{+}, \vec{p}_{\perp}\right), b_{s^{\prime}}^{\dagger}\left(p^{\prime+}, \vec{p}_{\perp}^{\prime}\right)\right\}=\left\{d_{s}\left(p^{+}, \vec{p}_{\perp}\right), d_{s^{\prime}}^{\dagger}\left(p^{\prime+}, \vec{p}_{\perp}^{\prime}\right)\right\}=(2 \pi)^{3} 2 p^{+} \delta\left(p^{+}-p^{\prime+}\right) \delta^{2}\left(\vec{p}_{\perp}-\vec{p}_{\perp}^{\prime}\right) \delta_{s s^{\prime}} .
$$

Then the LFWF representation of the electromagnetic vertex is,

$$
\begin{aligned}
\Gamma_{\lambda^{\prime} \lambda}^{\mu}\left(p, p^{\prime}\right)= & \sum_{s, \bar{s}} \int_{0}^{1} \frac{\mathrm{d} x}{2 x(1-x)} \int \frac{\mathrm{d}^{2} k_{\perp}}{(2 \pi)^{3}} \frac{1}{x^{\prime}} \sum_{s^{\prime}} \bar{u}_{s^{\prime}}\left(x^{\prime} p^{\prime+}, \vec{k}_{\perp}^{\prime}+x^{\prime} \vec{p}_{\perp}^{\prime}\right) \gamma^{\mu} u_{s}\left(x p^{+}, \vec{k}_{\perp}+x \vec{p}_{\perp}\right) \\
& \times \psi_{s^{\prime} \bar{s} / h}^{\left(\lambda^{\prime}\right) *}\left(x^{\prime}, \vec{k}_{\perp}^{\prime}\right) \psi_{s \bar{s} / h}^{(\lambda)}\left(x, \vec{k}_{\perp}\right)+\cdots,
\end{aligned}
$$

where $x^{\prime} p^{\prime+}=x p^{+}+q^{+}$, and $\vec{k}_{\perp}+x^{\prime} \vec{p}_{\perp}^{\prime}=\vec{k}_{\perp}+x \vec{p}_{\perp}+\vec{q}_{\perp}$. The dots represent the higher Fock sector contributions. Here for mesons with quark and antiquark having the same flavor, we have coupled the photon only to the quark. Otherwise, the form factor is exactly zero due to charge conjugation symmetry.

We introduce a boost invariant $t^{5}$ :

$$
\vec{\Delta}_{\perp}=\vec{q}_{\perp}-z \vec{p}_{\perp}^{\prime}=p^{+}\left(\frac{\vec{p}_{\perp}^{\prime}}{p^{\prime+}}-\frac{\vec{p}_{\perp}}{p^{+}}\right)
$$

where $z=q^{+} / p^{\prime+}$ is another boost invariant introduced above [see Eq. (1)]. Using $z$ and $\Delta_{\perp}$,

$$
x^{\prime}=x+z(1-x), \quad \vec{k}_{\perp}^{\prime}=\vec{k}_{\perp}+(1-x) \vec{\Delta}_{\perp} .
$$

The momentum fraction in the valence LFWF is constrained by $0 \leq x \leq 1$. Therefore, we only have access to the kinematical region $0 \leq z<1$. Negative $z$ probes sea quark contributions in higher Fock sectors. $z>1$ probes the timelike region, which is a different process in light-front dynamics. The Lorentz invariant momentum transfer squared $q^{2}=\left(p^{\prime}-p\right)^{2}$ depends on these two boost invariants,

$$
q^{2}=-\frac{z^{2} M_{h}^{2}+\Delta_{\perp}^{2}}{1-z} \equiv-Q^{2},
$$

where $M_{h}$ is the mass eigenvalue of the meson, i.e. $p^{2}=$ $p^{\prime 2}=M_{h}^{2}$. For the available kinematic range $(0 \leq z<1)$, $q^{2} \leq 0$, i.e. $q^{2}$ is spacelike. We introduce two special frames:

(I) transverse frame $(z=0)$, also known as Drell-Yan frame $\left(q^{+}=0\right): q^{2}=-\Delta_{\perp}^{2}=-q_{\perp}^{2}$

(II) longitudinal frame $\left(\Delta_{\perp}=0\right): q^{2}=-z^{2} M_{h}^{2} /(1-z)$. Our definition of the longitudinal frame is very similar to the longitudinal frame $\left(\vec{q}_{\perp}=0\right)$ introduced in the literature

\footnotetext{
${ }^{5}$ Note that $\vec{q}_{\perp}$, in general, is not a boost invariant.
}

$[12,14,18,19]$. However, $\vec{\Delta}_{\perp}$ is boost invariant while $\vec{q}_{\perp}$ is not. As we have mentioned earlier, the form factor in light-front dynamics depends on two boost invariants $z$ and $\Delta_{\perp}$, instead of one Lorentz invariant $Q^{2}$. This dependence is referred to as the frame dependence. Note that each pair of $\left(z, \Delta_{\perp}\right)$ denotes infinitely many reference frames related by light-front boost, longitudinal and transverse, as well as by rotation in the transverse plane.

The LFWF representation of the charge form factor is,

$$
\begin{aligned}
F\left(z, Q^{2}\right)= & \frac{\sqrt{1-z}}{1-\frac{1}{2} z} \sum_{s, \bar{s}} \int_{0}^{1} \frac{\mathrm{d} x}{2 x(1-x)} \int \frac{\mathrm{d}^{2} k_{\perp}}{(2 \pi)^{3}} \sqrt{\frac{x}{x+z(1-x)}} \\
& \times \psi_{s \bar{s} / h}^{*}\left(x+z(1-x), \vec{k}_{\perp}+(1-x) \vec{\Delta}_{\perp}\right) \psi_{s \bar{s} / h}\left(x, \vec{k}_{\perp}\right) \\
& +\cdots
\end{aligned}
$$

where $Q^{2}=\left(z^{2} M_{h}^{2}+\Delta_{\perp}^{2}\right) /(1-z)$. At $Q \rightarrow 0, z \rightarrow 0$ and $\Delta_{\perp} \rightarrow 0$ and $F\left(z, Q^{2}\right) \rightarrow 1$. At large $Q$, either large $\Delta_{\perp}$ or $z \rightarrow 1, F\left(z, Q^{2}\right) \rightarrow 0$. The explicit expression including higher Fock sectors is presented in Appendix A. In the Drell-Yan frame $\left(z=0, Q^{2}=\Delta_{\perp}^{2}\right)$, we obtain the familiar expression

$$
\begin{aligned}
F_{\mathrm{DY}}\left(Q^{2}\right)= & \sum_{s, \bar{s}} \int_{0}^{1} \frac{\mathrm{d} x}{2 x(1-x)} \int \frac{\mathrm{d}^{2} k_{\perp}}{(2 \pi)^{3}} \\
& \times \psi_{s \bar{s} / h}^{*}\left(x, \vec{k}_{\perp}+(1-x) \vec{\Delta}_{\perp}\right) \psi_{s \bar{s} / h}\left(x, \vec{k}_{\perp}\right) \\
& +\cdots
\end{aligned}
$$

In the longitudinal frame $\left(\Delta_{\perp}=0, Q^{2}=z^{2} M_{h}^{2} /(1-z)\right)$,

$$
\begin{aligned}
F_{\text {long }}\left(Q^{2}\right)= & \frac{\sqrt{1-z}}{1-\frac{1}{2} z} \sum_{s, \bar{s}} \int_{0}^{1} \frac{\mathrm{d} x}{2 x(1-x)} \int \frac{\mathrm{d}^{2} k_{\perp}}{(2 \pi)^{3}} \sqrt{\frac{x}{x+z(1-x)}} \\
& \times \psi_{s \bar{s} / h}^{*}\left(x+z(1-x), \vec{k}_{\perp}\right) \psi_{s \bar{s} / h}\left(x, \vec{k}_{\perp}\right)+\cdots
\end{aligned}
$$




\section{APPLICATION TO HEAVY QUARKONIA}

Recently, we proposed a model for heavy quarkonia based on light-front holographic QCD [35] and one-gluon exchange [36]. The theory is solved in the basis function approach (BLFQ, [37,38]). The one-gluon exchange implements the short-distance physics and supplies the proper spin structure. The resulting LFWFs have been used to compute a number of observables as well as in diffractive vector meson production, showing reasonable agreement with the available experimental data $[39,40]$. In this model, the violation of Lorentz symmetry leads to the spread of mass eigenvalues with the same angular momentum $j$ but different magnetic projection $m_{j}$. However, such violation is sufficiently small that it does not interfere with spectrum reconstruction. Therefore, it is interesting to see whether the frame dependence, also originating from the violation of the rotational symmetry, is under control.

As mentioned, the model is solved in a basis function approach. The LFWFs read,

$\psi_{s \bar{s}}\left(x, \vec{k}_{\perp}\right)=\sum_{n, m, l} \psi(n, m, l, s, \bar{s}) \phi_{n m}\left(\vec{k}_{\perp} / \sqrt{x(1-x)}\right) X_{l}(x)$.
Here $\phi_{n m}$ and $X_{l}$ are known analytic functions (see Refs. [37,38] for details). The basis space is truncated by $2 n+|m|+1 \leq N_{\max }$, and $l \leq L_{\max }$, and in the calculation, $N_{\max }=L_{\max }$ is chosen. The truncation introduces a UV scale $\Lambda_{\mathrm{UV}} \approx \kappa \sqrt{N_{\max }}$ and a resolution in the longitudinal direction $\Delta x \approx L_{\max }^{-1}$, where $\kappa$ is the confining strength whose value is given in Ref. [38]. Since form factors in light-front dynamics are represented as the convolution of LFWFs, the variables $z, \Delta_{\perp}$ and $Q^{2}$ are only supported up to the basis resolutions: $Q^{2} \lesssim \kappa^{2} N_{\max }$ in the transverse direction and $Q^{2} \lesssim M_{h}^{2} L_{\max }$ in the longitudinal direction. Beyond these regimes, the LFWFs are dominated by the asymptotics of the basis included within the limited basis space.

Figure 3 shows numerical results for charmonia $\eta_{c}, \chi_{c 0}$, $\eta_{c}^{\prime}$ and their bottomonium counterparts $\eta_{b}, \chi_{b 0}, \eta_{b}^{\prime}$. A basis truncation $N_{\max }=L_{\max }=32$ is used. The solid curves represent the Drell-Yan frame while the dashed curves the longitudinal frame. The shaded areas represent all other frames as obtained numerically through a dense sampling of the $z$ and $\Delta_{\perp}$ parameter space. We observe that overall, the frame dependence is moderate for both systems. The frame dependence of bottomonia is also smaller than that
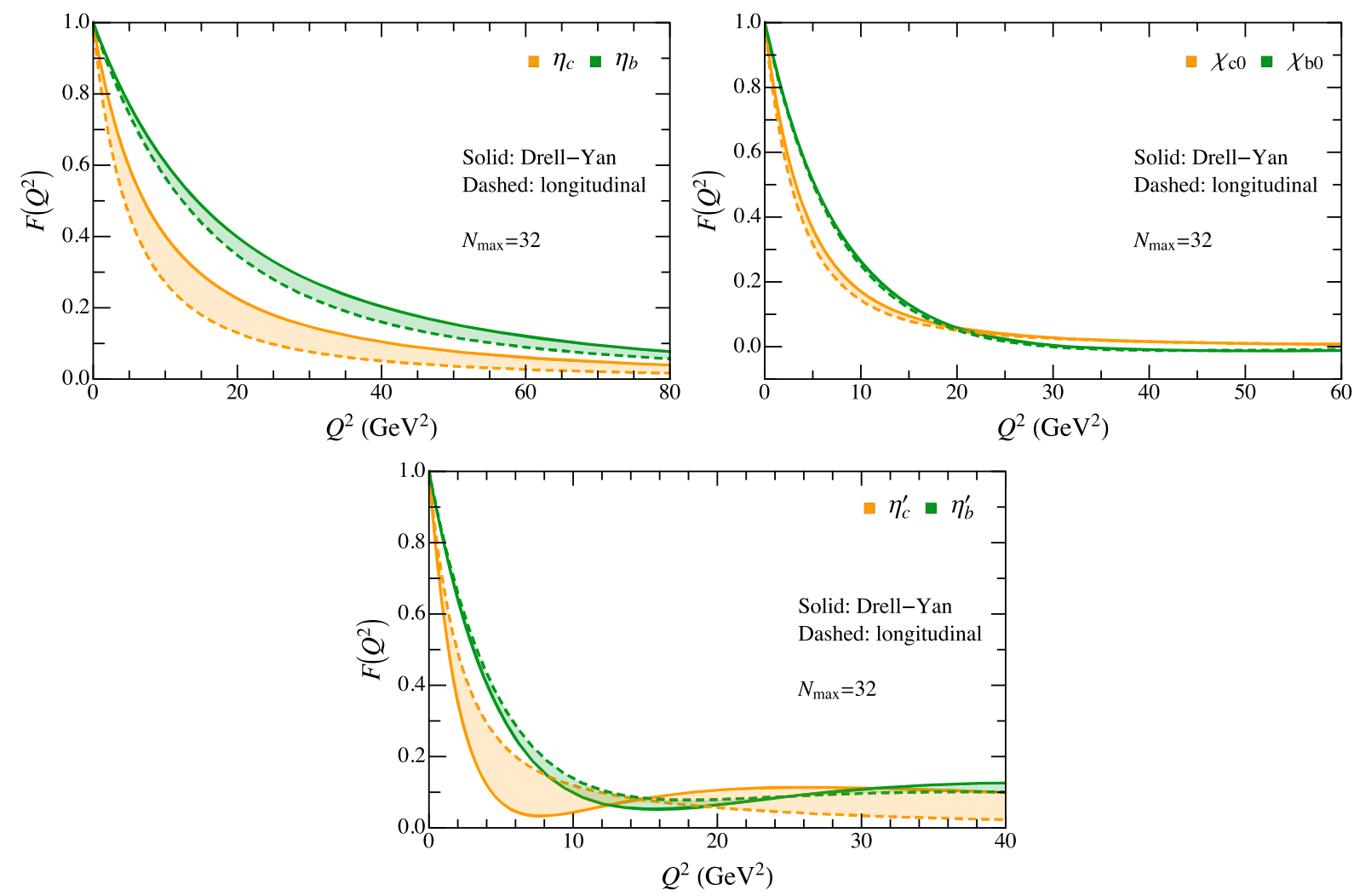

FIG. 3. Frame dependence of heavy quarkonia form factors. The solid curves represent the Drell-Yan frame while the dashed curves the longitudinal frame. The shaded areas represent all other frames as obtained numerically through a dense sampling of the $z$ and $\Delta_{\perp}$ parameter space. Note that the appearance of crossing lines in the third panel of may be misleading since there is a spread in the distribution of dense points nearby that are not visible at the resolution of the figure. The basis is truncated with $N_{\max }=L_{\max }=32$ (see text). 

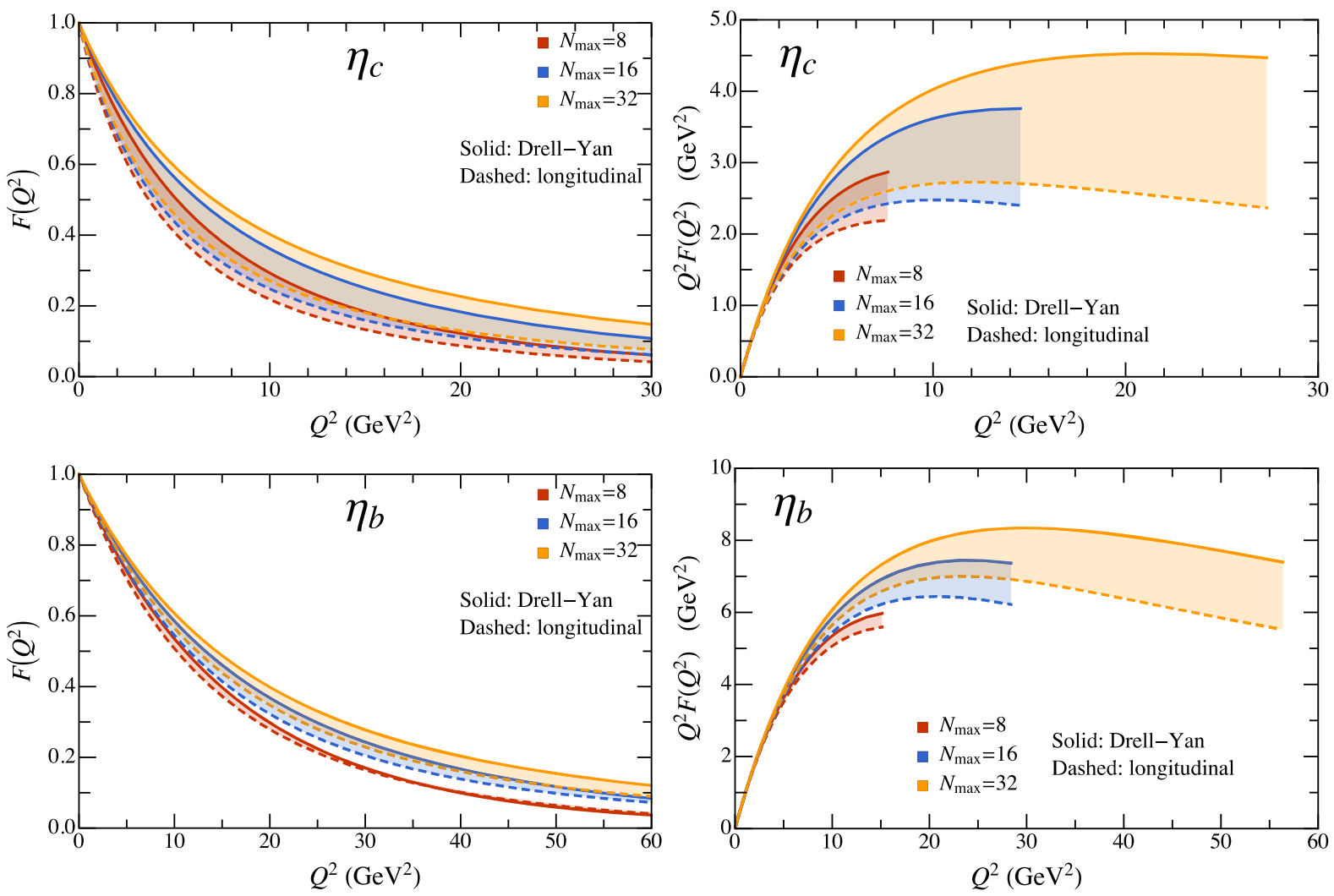

FIG. 4. Form factors with different basis truncations. On the right panels, form factors are shown up to the UV scale $\Lambda_{\mathrm{UV}}=\kappa \sqrt{N_{\max }}$. Note that in the basis representation, this is a soft cutoff.

of charmonia, which is consistent with the fact that bottomonia are less relativistic. These results also show that the Drell-Yan frame and the longitudinal frame are indeed two special frames: their respective form factors typically signal the extreme of the form factor $F\left(z, Q^{2}\right)$ for a given $Q^{2}$ and $0 \leq z<1$. $^{6}$ Therefore, the difference between the Drell-Yan frame and the longitudinal frame can be used to approximately characterize the frame dependence.

In the LFWFs, there are two sources of Lorentz symmetry violation. One comes from the Fock sector truncation and the associated effective interaction. The other comes from the basis truncation. Form factors of $\eta_{c}, \eta_{b}$ from different basis truncations $\left(N_{\max }=L_{\max }=8\right.$, $16,32)$ are shown in Fig. 4 . The basis convergence is observed to be reasonable up to the UV limit specified by the basis cutoff. To further see the basis truncation effects, we compare the form factors evaluated from the leading basis function $\left(N_{\max }=L_{\max }=1\right)$ and the full diagonalization $\left(N_{\max }=L_{\max }=32\right)$ in Fig. 5. The basis function is the solution of the long-distance

\footnotetext{
${ }^{6}$ This is most of the cases but not all.
}

part, i.e. the light-front holographic QCD (LFHQCD), without the contributions from the one-gluon exchange interaction. Therefore, this is essentially a comparison between LFHQCD ${ }^{7}$ and BLFQ for a particular set of parameters. We find that the excited states are more sensitive to the basis truncation. That is, we find that the excited states require more basis functions to resolve the spatial structure of the excited state wave functions. On the other hand, the ground states are more sensitive to the Fock sector truncation and to the model for the effective interaction. For example, the ground-state BLFQ form factors show more frame dependence. This is because, compared with LFHQCD (i.e. $N_{\max }=L_{\max }=1$ ), BLFQ has an additional interaction, the one-gluon exchange, which, upon Fock sector truncation, introduces an additional source of Lorentz symmetry violation.

\footnotetext{
${ }^{7}$ In the LFHQCD description of heavy quarkonia, a longitudinal function is required. Reference [38] compares two popular longitudinal functions and found they are almost identical for heavy quarkonia.
} 

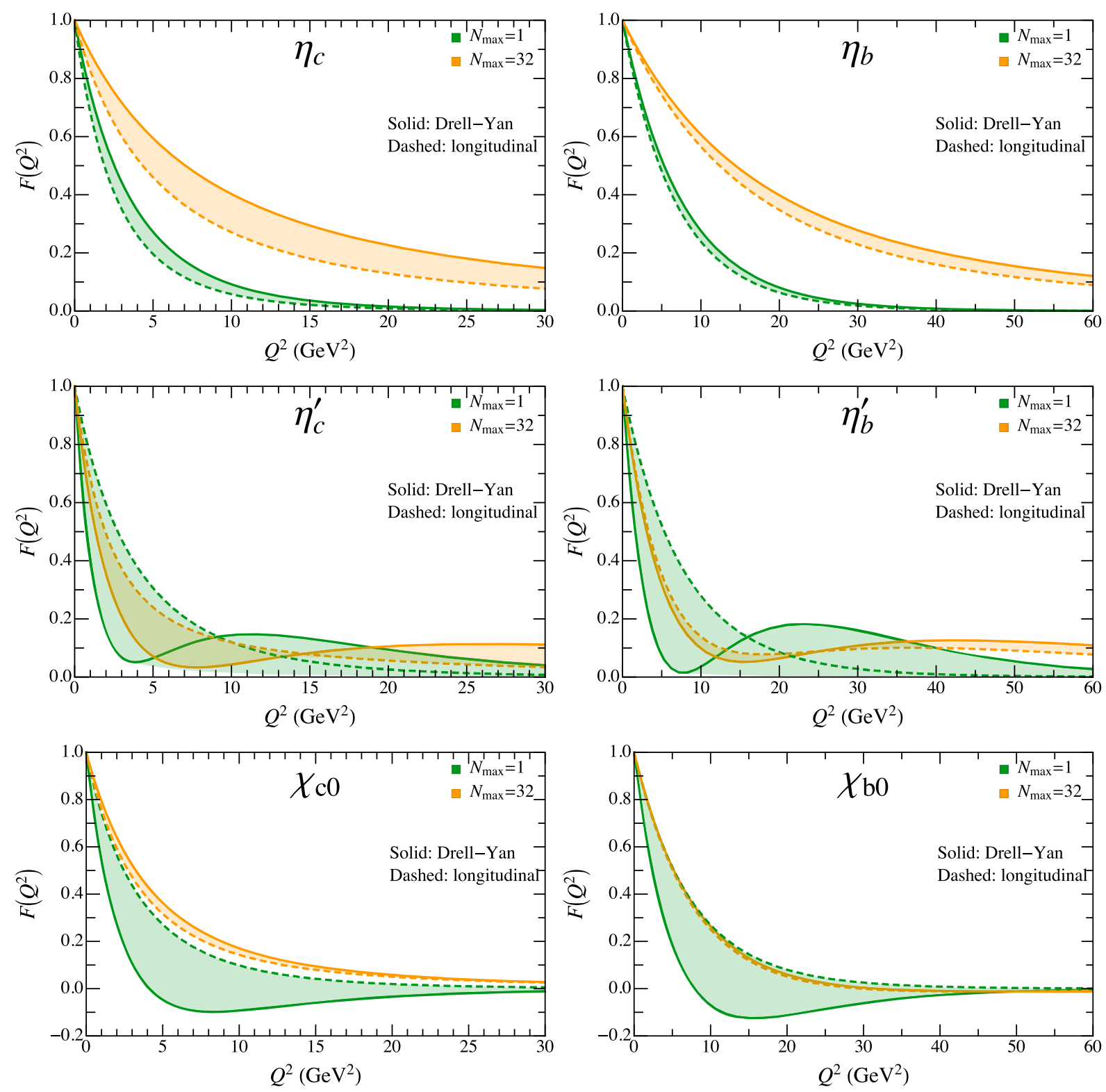

FIG. 5. Comparison of form factors from the leading basis function $\left(N_{\max }=L_{\max }=1\right)$ and BLFQ $\left(N_{\max }=L_{\max }=32\right)$.

\section{DISCUSSION AND CONCLUSIONS}

We have shown, using phenomenological LFWFs, scalar and pseudoscalar heavy quarkonia form factors admit moderate frame dependence. This dependence decreases from charmonium to bottomonium. We are therefore led to consider the frame dependence in light mesons. However, a similar model for light mesons is yet to be developed. As mentioned above, the phenomenological model is based on LFHQCD, whose LFWFs can be readily used for light mesons. On the other hand, using similar phenomenological wave functions, Isgur and Smith found that the pion form factor has a large frame dependence [14]. In Ref. [18], using a BSA, the discrepancy is attributed to the zero-mode contributions in the longitudinal frame. Such contributions are absent in the Drell-Yan frame.

Figure 6 presents the $\pi, \rho, \eta_{s}$ and $\phi$ form factors obtained from LFHQCD. ${ }^{8}$ Note that the spin effect is ignored in LFHQCD (cf. [41-45]). So $\rho$ and $\pi$ share the same spatial LFWF hence form factors in the Drell-Yan frame ${ }^{9}$

\footnotetext{
${ }^{8}$ Here $\eta_{s}$ is the ground-state pseudoscalar $s \bar{s}$ meson, which does not have any correspondence in Nature. The physical pseudoscalars $\eta$ and $\eta^{\prime}$ are dominated by the axial anomaly, which is not described in LFHQCD. $\eta_{s}$ is used as a theoretical construction in LFHQCD with a predicted mass $676 \mathrm{MeV}$.

${ }^{9}$ The longitudinal form factor depends on the physical mass.
} 

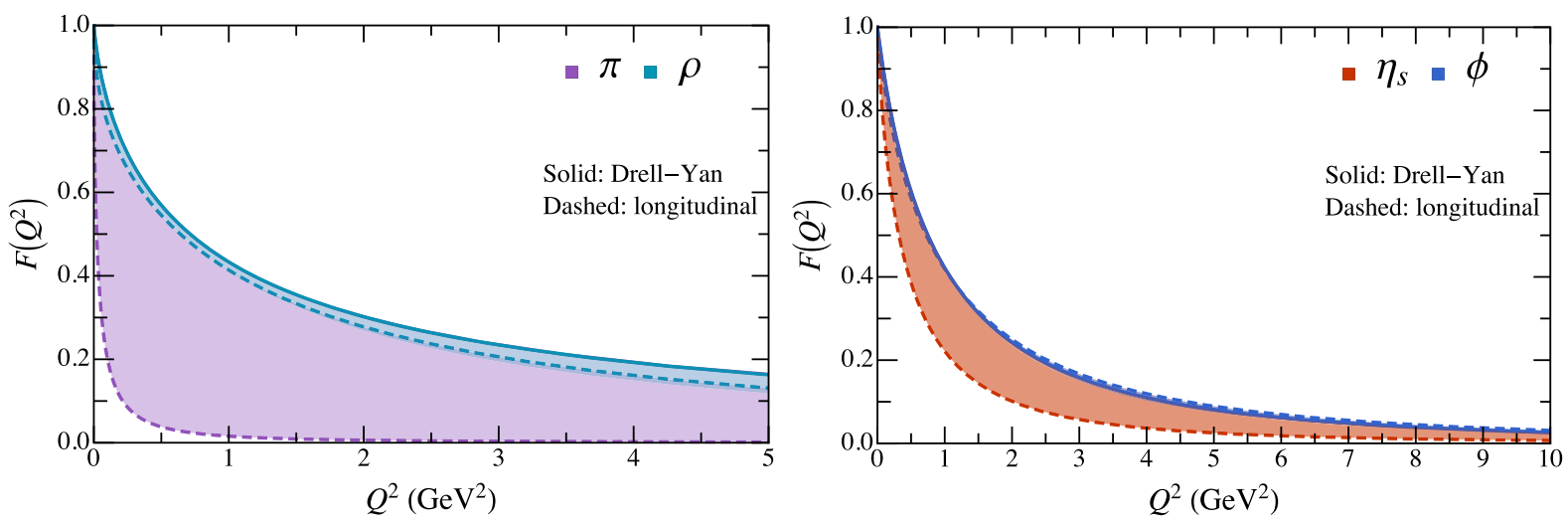

FIG. 6. Frame dependence of (left) $\pi, \rho$ and (right) $\eta_{s}, \phi$ form factors from LFHQCD. We adopt quark mass $m_{q}=46 \mathrm{MeV}$, $m_{s}=356 \mathrm{MeV}$ and confining scale $\kappa=0.54 \mathrm{GeV}$ [35]. The solid curves represent form factors in the Drell-Yan frame, which are the same for $\pi$ and $\rho$ (left) as well as $\eta_{s}$ and $\phi$ (right) in LFHQCD. The dashed curves are the form factors in the longitudinal frame. While both pseudoscalars display considerable frame dependence, that of $\pi$ seems especially large.

Similarly, $\phi$ and $\eta_{s}$ share the same LFWF hence form factors in the Drell-Yan frame. These light vector mesons $\rho, \phi$ show relatively small frame dependence in charge form factors as compared to the pseudoscalar mesons. The frame dependence in pion is especially large. Comparing $\rho$ and $\pi$, this large frame dependence can be attributed to the mass, as this is the only difference between $\rho$ and $\pi$ in LFHQCD (spin is assigned, not dynamical).

The mean-square radius $r_{\pi}^{2}$ controls the slope of the form factor at $Q^{2} \rightarrow 0$. It can be shown that, in the DrellYan frame, $r_{\pi}^{2} \propto \kappa^{-2} \ln M_{\pi}^{2} / \kappa^{2}+O\left(M_{\pi}^{2} / \kappa^{2}\right)$, whereas in the longitudinal frame, $r_{\pi}^{2} \propto \kappa^{2} / M_{\pi}^{4}+O\left(M_{\pi}^{2} / \kappa^{2}\right)$ up to a $\log$ correction $\ln M_{\pi} / \kappa$. Here $\kappa$ is the confining scale parameter. As $M_{\pi} \ll \kappa$, the discrepancy in $r_{\pi}^{2}$ explains the large frame dependence of the pion form factor at low $Q^{2}$. The asymptotics of $r_{\pi}^{2}$ obtained in the Drell-Yan frame is in agreement with the prediction from chiral perturbation theory $[46,47]$. Therefore, this large frame dependence points to the (lack of) chiral symmetry breaking in valence sector pion wave function, in particular, in the longitudinal direction. Chiral symmetry breaking on the light front is generally understood to require zero modes [48-51]. The conventional wisdom from the BSA suggest that this discrepancy may be caused by the (lack of) zero-mode contributions in the longitudinal frame, which have been omitted $[12,18]$. Of course, without a corresponding BSA, it is not clear how to take such contributions into account. Therefore, developing a light-front model implementing dynamical chiral symmetry breaking is essential for a self-consistent description of the pion.

To summarize, in this work, we develop a boostinvariant representation of space-like form factors in a general frame. We investigated the frame dependence of form factors in light-front dynamics. Heavy quarkonia are used as a concrete example. We show that the frame dependence is suppressed by the heavy quark mass. We identify the Drell-Yan frame and the longitudinal frame as two special frames, whose difference can be used to represent the frame dependence.

\section{ACKNOWLEDGMENTS}

The authors thank Alexander V. Smirnov, Stan Brodsky, Meijian Li, Christian Weiss, Raza Sufian and Tianbo Liu for valuable discussions. This work was supported in part by the U.S. Department of Energy (DOE) under Grants No. DE-FG02-87ER40371, No. DE-FG02-04ER41302, No. DE-SC0018223 (SciDAC-4/NUCLEI) and No. DESC0015376 (DOE Topical Collaboration in Nuclear Theory for Double-Beta Decay and Fundamental Symmetries). A portion of the computational resources were provided by the National Energy Research Scientific Computing Center (NERSC), which is supported by the U.S. DOE Office of Science.

\section{APPENDIX: LIGHT-FRONT WAVE FUNCTION REPRESENTATION BEYOND THE VALENCE SECTOR}

In this section, we present the general LFWF representation of the elastic form factors in a general frame with a boost-invariant parametrization using $z$ and $\vec{\Delta}_{\perp}$ $(0 \leq z \leq 1)$. The form factor admits a diagonal piece [Fig. 1(a)] and an off-diagonal piece [Fig. 1(b)]:

$$
F\left(z, Q^{2}\right)=F_{\text {diag }}\left(z, Q^{2}\right)+F_{\text {offdiag }}\left(z, Q^{2}\right)
$$

The diagonal part reads, 


$$
F_{\text {diag }}\left(z, Q^{2}\right)=\frac{2}{2-z} \sum_{n} \int\left[\mathrm{d} x_{i} \mathrm{~d}^{2} k_{i \perp}\right]_{n} \sum_{f} q_{f} \sqrt{\frac{x_{f}}{x_{f}^{\prime}}} \psi_{n}^{*}\left(\left\{x_{i}, \vec{k}_{i \perp}, \lambda_{i}\right\}\right) \psi_{n}\left(\left\{x_{i}^{\prime}, \vec{k}_{i \perp}^{\prime}, \lambda_{i}\right\}_{f}\right)
$$

where $x_{f}$ is the momentum fraction of the struck parton and $q_{f}$ its charge number. $\left\{x_{i}, \vec{k}_{i \perp}, \lambda_{i}\right\}=$ $\left(x_{1}, \vec{k}_{1 \perp}, \lambda_{1}, x_{2}, \vec{k}_{2 \perp}, \lambda_{2}, \ldots, x_{n}, \vec{k}_{n \perp}, \lambda_{n}\right)$ is a collection of parton quantum numbers for the $n$-body Fock sector. $\left\{x_{i}^{\prime}, \vec{k}_{i \perp}^{\prime}, \lambda_{i}^{\prime}\right\}_{f}$ is similar, except that a subscript $f$ is used to indicate the dependence on the choice of struck parton.

$$
x_{i}^{\prime}=\left\{\begin{array}{ll}
x_{i}(1-z), & \text { spectator; } \\
x_{i}(1-z)+z, & \text { struck parton. }
\end{array} \quad \vec{k}_{i \perp}^{\prime}= \begin{cases}\vec{k}_{i \perp}-x_{i} \vec{\Delta}_{\perp}, & \text { spectator; } \\
\vec{k}_{i \perp}+\left(1-x_{i}\right) \vec{\Delta}_{\perp}, & \text { struck parton. }\end{cases}\right.
$$

$\left[\mathrm{d} x_{i} \mathrm{~d}^{2} k_{i \perp}\right]_{n}$ is the $n$-body phase space integration measure:

$$
\int\left[\mathrm{d} x_{i} \mathrm{~d}^{2} k_{i \perp}\right]_{n}=S_{n}^{-1} \prod_{i=1}^{n} \sum_{\lambda_{i}} \int \frac{\mathrm{d} x_{i} \mathrm{~d}^{2} k_{i \perp}}{2 x_{i}(2 \pi)^{3}} 2(2 \pi)^{3} \delta\left(\sum_{i} x_{i}-1\right) \delta^{2}\left(\sum_{i} \vec{k}_{i \perp}\right) .
$$

In the Drell-Yan frame $(z=0)$, the diagonal part reduces to the Drell-Yan-West formula $[10,11]$.

The off-diagonal part reads,

$$
\begin{aligned}
F_{\text {offdiag }}\left(z, Q^{2}\right)= & \frac{2}{(2-z)} \sum_{n} \int\left[\mathrm{d} x_{i} \mathrm{~d}^{2} k_{i \perp}\right]_{n} \sum_{f} q_{f} \sum_{\lambda_{f}} \int_{0}^{1} \frac{\mathrm{d} \xi}{2 \xi(1-\xi)} \int \frac{\mathrm{d}^{2} k_{\perp}}{(2 \pi)^{3}} \\
& \times \sqrt{\xi(1-\xi)} \psi_{n}^{*}\left(\left\{x_{i}, \vec{k}_{i \perp}, \lambda_{i}\right\}\right) \psi_{n+2}\left(\left\{x_{i}^{\prime}, \vec{k}_{i \perp}^{\prime}, \lambda_{i}^{\prime}\right\}_{f}\right)
\end{aligned}
$$

where $x_{f}$ is the momentum fraction of the struck parton and $q_{f}$ its charge number, $\left[\mathrm{d} x_{i} \mathrm{~d}^{2} k_{i \perp}\right]_{n}$ is again the $n$-body phase space integration measure. The collections of parton quantum numbers $\left\{x_{i}, \vec{k}_{i \perp}, \lambda_{i}\right\}$ and $\left\{x_{i}^{\prime}, \vec{k}_{i \perp}^{\prime}, \lambda_{i}^{\prime}\right\}_{f}$ are similar to the diagonal part, except that, now, it is understood the spectators within the initial and final states pair up.

$$
\begin{gathered}
x_{i}^{\prime}=\left\{\begin{array}{ll}
x_{i}(1-z), & \text { spectator; } \\
z \xi, & \text { struck quark; } \\
z(1-\xi), & \text { struck antiquark. }
\end{array} \quad \vec{k}_{i \perp}^{\prime}= \begin{cases}\vec{k}_{i \perp}-x_{i} \vec{\Delta}_{\perp}, & \text { spectator; } \\
\vec{k}_{\perp}+\xi \vec{\Delta}_{\perp}, & \text { struck quark; } \\
-\vec{k}_{\perp}+(1-\xi) \vec{\Delta}_{\perp}, & \text { struck antiquark. }\end{cases} \right. \\
\lambda_{i}^{\prime}= \begin{cases}\lambda_{i}, & \text { spectator; } \\
\lambda_{f}, & \text { struck quark; } \\
-\lambda_{f}, & \text { struck antiquark. }\end{cases}
\end{gathered}
$$

In the Drell-Yan frame $(z=0)$, the off-diagonal part has only zero-mode contributions.

[1] R. J. Perry, Hamiltonian light front field theory and quantum chromodynamics, arXiv:hep-th/9407056.

[2] W. M. Zhang, Light front dynamics and light front QCD, Chin. J. Phys. 32, 717 (1994).

[3] M. Burkardt, Light front quantization, Adv. Nucl. Phys. 23, 1 (1996).

[4] A. Harindranath, An introduction to light front dynamics for pedestrians, arXiv:hep-ph/9612244.

[5] S. J. Brodsky, H. C. Pauli, and S. S. Pinsky, Quantum chromodynamics and other field theories on the light cone, Phys. Rep. 301, 299 (1998).

[6] J. Carbonell, B. Desplanques, V. A. Karmanov, and J. F. Mathiot, Explicitly covariant light front dynamics and relativistic few body systems, Phys. Rep. 300, 215 (1998).
[7] J. R. Hiller, Nonperturbative light-front Hamiltonian methods, Prog. Part. Nucl. Phys. 90, 75 (2016).

[8] S. D. Drell and T. M. Yan, Connection of Elastic Electromagnetic Nucleon Form-Factors at Large $Q^{2}$ and Deep Inelastic Structure Functions Near Threshold, Phys. Rev. Lett. 24, 181 (1970).

[9] G. B. West, Phenomenological Model for the Electromagnetic Structure of the Proton, Phys. Rev. Lett. 24, 1206 (1970).

[10] S. J. Brodsky, R. Roskies, and R. Suaya, Quantum electrodynamics and renormalization theory in the infinite momentum frame, Phys. Rev. D 8, 4574 (1973).

[11] S. J. Brodsky and S. D. Drell, The anomalous magnetic moment and limits on fermion substructure, Phys. Rev. D 22, 2236 (1980). 
[12] S. J. Brodsky and D. S. Hwang, Exact light cone wave function representation of matrix elements of electroweak currents, Nucl. Phys. B543, 239 (1999).

[13] S. S. Chabysheva and J. R. Hiller, A light-front coupledcluster method for the nonperturbative solution of quantum field theories, Phys. Lett. B 711, 417 (2012).

[14] N. Isgur and C. H. Llewellyn Smith, The applicability of perturbative QCD to exclusive processes, Nucl. Phys. B317, 526 (1989).

[15] Y. Li, V. A. Karmanov, P. Maris, and J. P. Vary, Nonperturbative calculation of the scalar Yukawa theory in four-body truncation, Few-Body Syst. 56, 495 (2015).

[16] Y. Li, V. A. Karmanov, P. Maris, and J. P. Vary, Ab initio approach to the non-perturbative scalar Yukawa model, Phys. Lett. B 748, 278 (2015).

[17] V. A. Karmanov, Y. Li, A. V. Smirnov, and J. P. Vary, Nonperturbative solution of scalar Yukawa model in twoand three-body Fock space truncations, Phys. Rev. D 94, 096008 (2016).

[18] M. Sawicki, Soft charge form-factor of the pion, Phys. Rev. D 46, 474 (1992).

[19] S. Simula, Comparison among Hamiltonian light front formalisms at $\mathrm{q}+=0$ and $\mathrm{q}+$ not equal to 0: Space-like elastic form-factors of pseudoscalar and vector mesons, Phys. Rev. C 66. 035201 (2002).

[20] D. Melikhov and S. Simula, End point singularities of Feynman graphs on the light cone, Phys. Lett. B 556, 135 (2003).

[21] H. M. Choi and C. R. Ji, Nonvanishing zero modes in the light front current, Phys. Rev. D 58, 071901 (1998).

[22] C. S. Mello, J.P. B.C. de Melo, and T. Frederico, Minkowski space pion model inspired by lattice QCD running quark mass, Phys. Lett. B 766, 86 (2017).

[23] P. Maris and P. C. Tandy, The quark photon vertex and the pion charge radius, Phys. Rev. C 61, 045202 (2000).

[24] J. H. O. Sales, T. Frederico, B. V. Carlson, and P. U. Sauer, Light front Bethe-Salpeter equation, Phys. Rev. C 61, 044003 (2000).

[25] V. A. Karmanov and J. Carbonell, Solving Bethe-Salpeter equation in Minkowski space, Eur. Phys. J. A 27, 1 (2006).

[26] K. Kusaka and A. G. Williams, Solving the Bethe-Salpeter equation for scalar theories in Minkowski space, Phys. Rev. D 51, 7026 (1995).

[27] C. Gutierrez, V. Gigante, T. Frederico, G. Salm, M. Viviani, and L. Tomio, Bethe-Salpeter bound-state structure in Minkowski space, Phys. Lett. B 759, 131 (2016).

[28] J. Carbonell, T. Frederico, and V. A. Karmanov, Euclidean to Minkowski Bethe-Salpeter amplitude and observables, Eur. Phys. J. C 77, 58 (2017).

[29] J. Carbonell, T. Frederico, and V. A. Karmanov, Bound state equation for the Nakanishi weight function, Phys. Lett. B 769, 418 (2017).

[30] W. de Paula, T. Frederico, G. Salm, and M. Viviani, Advances in solving the two-fermion homogeneous Bethe-Salpeter equation in Minkowski space, Phys. Rev. D 94, 071901 (2016).

[31] W. de Paula, T. Frederico, R. Pimentel, G. Salm, and M. Viviani, Fermionic bound states in Minkowski-space:
Light-cone singularities and structure, Eur. Phys. J. C 77, 764 (2017).

[32] M.-j. Li, Y. Li, P. Maris, and J. P. Vary (to be published).

[33] H. Y. Cheng, C. Y. Cheung, and C. W. Hwang, Mesonic form-factors and the Isgur-Wise function on the light front, Phys. Rev. D 55, 1559 (1997).

[34] C. R. Ji and C. Mitchell, Frame independence of exclusive amplitudes in the light front quantization, arXiv:hep-ph/0003329.

[35] S. J. Brodsky, G. F. de Teramond, H. G. Dosch, and J. Erlich, Light-front holographic QCD and emerging confinement, Phys. Rep. 584, 1 (2015).

[36] P. Wiecki, Y. Li, X. Zhao, P. Maris, and J. P. Vary, Basis light-front quantization approach to positronium, Phys. Rev. D 91, 105009 (2015).

[37] Y. Li, P. Maris, X. Zhao, and J. P. Vary, Heavy quarkonium in a holographic basis, Phys. Lett. B 758, 118 (2016).

[38] Y. Li, P. Maris, and J. P. Vary, Quarkonium as a relativistic bound state on the light front, Phys. Rev. D 96, 016022 (2017).

[39] J. P. Vary, L. Adhikari, G. Chen, Y. Li, P. Maris, and X. Zhao, Basis light-front quantization: Recent progress and future prospects, Few-Body Syst. 57, 695 (2016).

[40] G. Chen, Y. Li, P. Maris, K. Tuchin, and J. P. Vary, Diffractive charmonium spectrum in high energy collisions in the basis light-front quantization approach, Phys. Lett. B 769, 477 (2017).

[41] M. Ahmady, F. Chishtie, and R. Sandapen, Spin effects in the pion holographic light-front wavefunction, Phys. Rev. D 95, 074008 (2017).

[42] O. Leitner, J.-F. Mathiot, and N. A. Tsirova, The pion wave function in covariant light-front dynamics, Eur. Phys. J. A 47, 17 (2011).

[43] G. H. S. Yabusaki, I. Ahmed, M. A. Paracha, J. P. B. C. de Melo, and B. El-Bennich, Pseudoscalar mesons with symmetric bound state vertex functions on the light front, Phys. Rev. D 92, 034017 (2015).

[44] C. S. Mello, J. P. B. C. de Melo, and T. Frederico, The pion electromagnetic structure with self-energy, J. Phys. Conf. Ser. 706, 052008 (2016).

[45] E. O. da Silva, J. P. B. C. de Melo, B. El-Bennich, and V. S. Filho, Pion and kaon elastic form factors in a refined lightfront model, Phys. Rev. C 86, 038202 (2012).

[46] M. A. B. Beg and A. Zepeda, Pion radius and isovector nucleon radii in the limit of small pion mass, Phys. Rev. D 6 , 2912 (1972).

[47] M. K. Volkov and V. N. Pervushin, The electromagnetic form-factor of the pion, Phys. Lett. 51B, 356 (1974).

[48] S. R. Beane, Broken chiral symmetry on a null plane, Ann. Phys. (Amsterdam) 337, 111 (2013).

[49] M. H. Wu and W. M. Zhang, Chiral symmetry in light front QCD, J. High Energy Phys. 04 (2004) 045.

[50] M. Burkardt and H. El-Khozondar, A $(3+1)$-dimensional light front model with spontaneous breaking of chiral symmetry, Phys. Rev. D 55, 6514 (1997).

[51] M. Burkardt, Dynamical vertex mass generation and chiral symmetry breaking on the light front, Phys. Rev. D 58, 096015 (1998). 